\title{
Maternal Distress during Pregnancy and Offspring Childhood Overweight
}

\author{
Katja Glejsted Ingstrup, ${ }^{1}$ Camilla Schou Andersen, ${ }^{2}$ Teresa Adeltoft Ajslev, ${ }^{2}$ \\ Pernille Pedersen, ${ }^{3}$ Thorkild I. A. Sørensen, ${ }^{2}$ and Ellen A. Nohr ${ }^{1}$ \\ ${ }^{1}$ Section for Epidemiology, Department of Public Health, Aarhus University, Bartholins Allé 2, 8000 Aarhus C, Denmark \\ ${ }^{2}$ Institute of Preventive Midicine, Copenhagen University Hospital, Øster Søgade 18, 1357 Copenhagen K, Denmark \\ ${ }^{3}$ Psychiatric Research Unit West, Regional Psychiatric Services, Gl. Landevej 43, 7400 Herning, Denmark
}

Correspondence should be addressed to Katja Glejsted Ingstrup, ki@soci.au.dk

Received 11 January 2012; Accepted 19 March 2012

Academic Editor: Devin Mann

Copyright (C) 2012 Katja Glejsted Ingstrup et al. This is an open access article distributed under the Creative Commons Attribution License, which permits unrestricted use, distribution, and reproduction in any medium, provided the original work is properly cited.

\begin{abstract}
Background. Maternal distress during pregnancy increases the intrauterine level of glucocorticoids, which may have long-term health consequences for the child. Objective. To examine if distress as a combined measure of anxiety, depression, and stress of the mother during pregnancy was associated with offspring childhood overweight at age 7. Methods. We performed a cohort study using prospective data from 37,764 women and child dyads from the Danish National Birth Cohort (1996-2002). At a telephone interview at approximately 30 weeks gestation, the women reported whether they felt anxious, depressed, or stressed. The 95 percentile for body mass index in an international reference defined childhood overweight at any given age. Logistic regression was used for the analyses. Results. The prevalence of overweight children at 7 years of age was 9.9\%. Prenatal exposure to maternal distress during pregnancy was not associated with childhood overweight at 7 years of age (adjusted OR 1.06 (95\% CI 0.96; 1.18)). In analyses stratified on sex, a small tendency of overweight was seen in boys (OR $1.15(0.99 ; 1.33)$ ), but not in girls (OR 0.98 (0.85; 1.13)). Conclusions. Maternal distress during pregnancy appeared to have limited, if any, influence on the risk of overweight in offspring at 7 years of age.
\end{abstract}

\section{Introduction}

Childhood overweight is a substantial problem among children. In 2003, the prevalence of overweight among Danish children aged $6-8$ years was $15 \%$ and $21 \%$ among boys and girls, respectively [1]. Ideally, the prevention of childhood overweight and obesity should begin as early as possible, which may even be before birth [2]. Changes to the intrauterine environment, caused by stress or malnutrition of the mother during pregnancy, may modify fetal metabolism by influencing regulatory hormonal pathways. Such disturbances may persist after fetal life and affect the growth and health of the child [2].

Psychosocial factors such as maternal distress during pregnancy, physiological or psychological, increase the release of glucocorticoids (cortisol) [3]. Cortisol may be passed on from the mother to the child through the placenta [4] and potentially affect the developmental processes of the child. Expecting mothers who reported higher levels of stress or who worried about their pregnancy were found to have higher levels of salivary cortisol measured in the evening [5]. Measures of maternal stress during pregnancy have been associated with later health of the child including the risk of offspring pediatric disease [6], cerebral palsy [7], astma (in boys only) [8], and type 1 diabetes, [9] but not with epilepsy [10] or autism [11]. Also maternal bereavement due to loss of a relative, either while pregnant or one year before conception, has also been linked to childhood overweight [12] but it is not known if this association is present at lower maternal stress levels. We investigated whether children of mothers, who felt anxious, depressed, stressed, or worried while pregnant, had a higher risk of overweight at 7 years of age. Since different hormonal changes during pregnancy may potentially trigger different expressions in boys and girls [13], we also took into account the sex of the child. 


\section{Methods}

2.1. Study Population. The study was based on the Danish National Birth Cohort (DNBC), which was established from 1996 to 2002 where 100,419 pregnancies were enrolled from a total of 92,276 mainly Scandinavian women. A description of the enrolment and design has previously been published [14].

Computerised telephone interviews at approximately gestational weeks 16 and 30 and when the child was 6 and 18 months old were used to obtain information about prenatal exposures, maternal health, use of medicine, lifestyle, and the health and development of the child [14]. The questionnaires to the women contained a specific protocol for the interviewer to follow to generate uniformity across the different interviewers. A 7-year follow up was completed in spring 2011 and consisted of a web-based or posted questionnaire about the health, lifestyle, and development of the child including weight and height. The parents received the questionnaire in the month of the child's 7th birthday.

For the present study, mothers and their offsprings were included if they had participated in the 7-year follow up $(n=53,838)$. We excluded children with missing data on height, weight, or date of height and weight measurement and children with more than 30 days between measurement of height and weight $(n=4,592)$. We also excluded twins and triplets $(n=828)$, and for mothers who participated with more than one child in the cohort, all other children than the firstborn within the study period were excluded $(n=4,814)$. Children born before gestational week $37(n=$ $1,723)$, children born to mothers with diabetes $(n=427)$, and children whose mothers had not participated in the early and late pregnancy interview $(n=3,690)$ were also excluded. The final study population consisted of 37,764 mother-child dyads. All participants provided informed written consent and the study was approved by all of the scientific ethics committees in Denmark and by the Danish National Data Protection Agency.

2.2. Maternal Distress. The mothers' report of feeling anxious, depressed, or stressed during pregnancy was considered the main exposure. It was based on nine questions (Table 1) from the second pregnancy interview in approximately gestational week 30 (interquartile range 29-33). Their answer related to the entire pregnancy experienced at that point in time. The six questions about anxiety and depression originated from the validated Symptoms Checklist-92 (SCL92) $[15,16]$ and had originally five answer categories but only three were used for the women in the DNBC. For each woman, a likert score was generated by summing the scores for each of the three questions for anxiety and depression ("not at all" = 0 , “a little" = 1 , and "a lot" = 2). The reliability of the questions was assessed using Cronbach alpha coefficients. For anxiety and depression the coefficients were 0.56 and 0.53 respectively. The three questions about stress originated from the validated General Health Questionnaire 60 (GHQ60) [17] with an original four answer categories where $0=$ "better than normal," 1 = "same as normal," $2=$ "worse than normal," and 3 = "much worse than normal."
TABLe 1: Questions about anxiety, depression, and stress during pregnancy*. "Have you...."

\begin{tabular}{lcc}
\hline Questions & Covering & $\begin{array}{c}\text { Items } \\
\text { taken from }\end{array}$ \\
\hline $\begin{array}{l}\text { felt frightened and anxious for any } \\
\text { reason? }\end{array}$ & Anxiety & SCL-92 \\
$\begin{array}{l}\text { felt nervous or at unease? } \\
\text { felt tense and exhausted? }\end{array}$ & Anxiety & SCL-92 \\
\hline $\begin{array}{l}\text { felt that the future looked hopeless? } \\
\text { felt sad or blue? }\end{array}$ & Depression & SCL-92 \\
felt that everything was a big effort? & Depression & SCL-92 \\
\hline $\begin{array}{l}\text { felt under a constant pressure? } \\
\text { been more touchy and quick-tempered } \\
\text { than usually? }\end{array}$ & SCL-92 \\
$\begin{array}{l}\text { felt that the demands on you were too } \\
\text { big? }\end{array}$ & Stress & GHQ-60 \\
\hline
\end{tabular}

GHQ-60; General Health Questionnaire 60, SCL-92; Symptoms Checklist 92 (26-28).

* From the second interview at approximately gestational week 30 .

It was therefore decided to generate a stress score as follows 1 = "not at all," 2 = "a little," and 3 = "a lot," which had a Cronbach alpha of 0.42 . A combined measure of distress was generated as a combined added score for all nine questions and had a Cronbach alpha of 0.74 . For all four variables, the women were divided into two groups according to the cut-off value closest to the 80th percentile thus women exceeding this cutoff will be referred to as feeling anxious, depressed or stressed. However, for anxiety, only $11.7 \%$ of the mothers belonged to the high exposure category, because a large group of women had a sum score of 2, which prevented us from using the 80th percentile to define the high exposure category. Therefore, being anxious was a less inclusive measurement than measures of depression and stress.

Maternal worrying was based on two questions: worrying about the birth or worrying about the unborn child. They were asked both in the early and late pregnancy interview and the mother was categorized as worried, only if she answered yes at both points in time. Support from surroundings regarded the mothers contact to family members by phone or in person with "every day" or "several times a week" categorized as often.

Socioeconomic status was based on the education and job situation of both the mother and the father and defined as the highest level within the couple. It was categorized in three groups: leaders and parents with higher education was categorized as "high," parents with intermediate length of education as "intermediate," and unemployed or uneducated parents as "low."

2.3. Childhood Overweight. For each child, the body mass index $(\mathrm{BMI})\left(\mathrm{kg} / \mathrm{m}^{2}\right)$ was calculated using the weight and height of the child, which were either measured by the parents, the general practitioner, or the school nurse. It was up to the parents to choose which earlier measured weight and height they would record in the questionnaire 
and therefore some chose measures taken by the general practitioner at the 5-year health examination. The age span of the children was 5-8 years with $80 \%$ being 7 years old. Childhood overweight, was defined by using the sex and agespecific BMI references proposed by Cole et al. [18], where the 95 percentiles at any given age were used as the cutoff point for overweight [18]. We grouped the children in intervals of 6 months. For 7 years of age the cut-off points for overweight were $17.92 \mathrm{~kg} / \mathrm{km}^{2}$ and $17.75 \mathrm{~kg} / \mathrm{km}^{2}$ for boys and girls, respectively.

2.4. Covariates. Additional factors associated with childhood overweight were chosen a-priori based on the available literature. Information about parity, maternal prepregnancy BMI, smoking and recreational exercise during pregnancy came from the early pregnancy interview. Information about gestational weight gain and duration of breastfeeding came from the interview 6 months postpartum. These and other variables were categorized according to Table 2 .

2.5. Statistical Methods. Firstly, we examined maternal characteristics according to maternal distress and childhood overweight by using the Chi-square test. Next, we used multiple logistic regression models to estimate odds ratios for the association between maternal distress and other psycho-social factors and overweight of the children at 7 years of age. In the first adjusted model, we controlled for; age, parity, prepregnancy BMI, smoking during pregnancy, and socioeconomic status. In a second adjusted analysis, we also controlled for breastfeeding, gestational weight gain and recreational exercise of the mother. For one of the distress variables (anxiety), we found a significant difference in overweight between boys and girls. We therefore added an interaction term to the model so that the results could also be shown separately for boys and girls. Results are presented with $95 \%$ confidence intervals and $P$ values below 0.05 were considered statistically significant. All analyses were carried out using the statistical computer programme STATA (Version 10 Stata Corp, 4905 Lakeway Drive, College Station, TX 77845, USA).

\section{Results}

Distress during pregnancy was reported in $12.3 \%$ of the mothers with $11.7 \%$ of them feeling anxious, $17.9 \%$ depressed, and $20.6 \%$ stressed. Mothers who were less than 25 years old, singles, smokers, or gained more than $20 \mathrm{~kg}$ during pregnancy were more likely to feel anxious or depressed. Mothers who had given birth before more often reported feeling depressed and stressed than primiparous mothers. Also, mothers in the lowest social group more often felt depressed.

The mean BMI of the children in the study population was mean 15.7 (SD 1.7), and the prevalence of overweight children was $9.9 \%, 8.7 \%$ in boys and $11.5 \%$ in girls. Mothers who were overweight or obese before pregnancy or had a large gestational weight gain more often had overweight children. Also, mothers of overweight children were slightly younger, more often multiparous, smokers, or of medium or low socioeconomic status. Further, they were less likely to exercise during pregnancy and they breastfed their children for at shorter period.

\subsection{Pregnancy Distress in Relation to Childhood Overweight.} In the adjusted analyses, we found no association between maternal distress during pregnancy and the risk of overweight in the child (OR $1.06(0.96 ; 1.18)$ ) (Table 3$)$. In boys, a modest increased risk of overweight was indicated (OR $1.15(0.99 ; 1.33))$ but not in girls (OR $0.98(0.85 ; 1.13)$ ). The same pattern was observed when analysing feelings of anxiety, depression, or stress separately. Adding adjustment for breastfeeding, gestational weight gain and recreational exercise of the mother to the model only let to minor changes in the estimates (results not shown).

A modest increased risk of childhood overweight was seen in children of mothers who worried during pregnancy about the birth or the health of the child (OR 1.10 $(1.00 ; 1.22))$ and estimates were similar in boys and girls. Lack of social support seemed to be slightly protective against childhood overweight (OR $0.93(0.82 ; 1.04))$ whereas children of mothers of low or medium socioeconomic status had an increased risk of overweight. Only in the analysis of anxiety did we find that the sex of the child seemed to modify the association with childhood overweight $(P=0.05)$.

\section{Discussion}

In this population of women, we did not find maternal distress during pregnancy to be clearly associated with childhood overweight in the offspring at 7 years of age. Neither did separate measures for maternal feelings of anxiety, depression, and stress support any association.

It was a biologically plausible hypothesis that maternal distress during pregnancy may cause childhood obesity due to alterations of the metabolism of the child (2-5). Studies investigating the associations between prenatal distress and childhood overweight are, however, scarce. In a recent study by $\mathrm{Li}$ et al. [12], maternal bereavement, due to loss of a child or husband, was associated with childhood overweight, and most strongly for losses happening before conception than during pregnancy. Losing a child or husband causes severe distress and sadness in a pregnant mother, whereas the levels of distress measured in our study were more commonly experienced feelings of anxiety, depression, and stress and not caused by an identified specific event. Moreover, the exposure contrast in our cohort may be relatively low due to the healthy nature of the women who were of higher socioeconomic status and had better outcomes than the general pregnant population in Denmark [19]. Thus, the relatively mild measurement of distress (emotional stress) compared to a more severe type of bereavement may play a role for the contrast of the exposure in this study. On the other hand, in the study by Li et al. [12], the association between bereavement and offspring overweight did not show up until the children were around 10 years of age. So, our finding of little association between distress during 
TABLE 2: Maternal distress during pregnancy covering anxiety, depression, and stress, and childhood overweight according to maternal characteristics.

\begin{tabular}{|c|c|c|c|c|c|c|c|}
\hline & \multicolumn{7}{|c|}{ Maternal mental health } \\
\hline & \multicolumn{2}{|l|}{ Total } & \multirow{2}{*}{$\begin{array}{c}\text { Maternal distress } \\
\% \\
\end{array}$} & \multirow{2}{*}{$\begin{array}{c}\text { Feeling anxious } \\
\qquad \% \\
\end{array}$} & \multirow{2}{*}{$\begin{array}{c}\text { Feeling depressed } \\
\%\end{array}$} & \multirow{2}{*}{$\begin{array}{l}\text { Feeling stressed } \\
\qquad \% \\
\end{array}$} & \multirow{2}{*}{$\begin{array}{c}\text { Childhood } \\
\text { overweight } \\
\% \\
\end{array}$} \\
\hline & $n$ & $\%$ & & & & & \\
\hline Total population & 37,764 & 100.0 & 12.3 & 11.7 & 17.9 & 20.6 & 9.9 \\
\hline \multicolumn{8}{|l|}{ Maternal age } \\
\hline$<25$ & 4,337 & 11.5 & 15.6 & 14.5 & 15.5 & 13.5 & 10.5 \\
\hline $25-28$ & 15,919 & 42.2 & 38.4 & 39.8 & 38.3 & 38.7 & 9.7 \\
\hline $39-34$ & 13,012 & 34.5 & 33.3 & 33.6 & 33.2 & 34.8 & 9.8 \\
\hline$\geq 35$ & 4,496 & 11.9 & 12.7 & 12.1 & 13.0 & 13.0 & 10.1 \\
\hline \multicolumn{8}{|l|}{ Marital status } \\
\hline Married/partner & 36,164 & 98.2 & 96.5 & 96.7 & 96.3 & 97.1 & 9.7 \\
\hline Single & 645 & 1.8 & 3.5 & 3.3 & 3.7 & 2.9 & 14.1 \\
\hline Missing & 955 & & & & & & \\
\hline \multicolumn{8}{|l|}{ Parity } \\
\hline Primiparous & 18,817 & 49.8 & 43.3 & 51.4 & 43.6 & 40.0 & 9.1 \\
\hline Multiparous & 18,947 & 50.2 & 56.7 & 48.6 & 56.4 & 60.0 & 10.7 \\
\hline \multicolumn{8}{|l|}{ Prepregnancy BMI } \\
\hline Underweight $<18,5$ & 1,523 & 4.1 & 4.5 & 4.2 & 4.5 & 4.5 & 3.7 \\
\hline Normal weight $18,5-24,9$ & 26,182 & 70.5 & 68.1 & 69.1 & 67.2 & 69.5 & 7.5 \\
\hline Overweight 25-29,9 & 6,924 & 18.6 & 20.2 & 19.4 & 20.9 & 19.1 & 15.3 \\
\hline Obese $\geq 30$ & 2,539 & 6.8 & 7.2 & 7.3 & 7.4 & 6.9 & 22.0 \\
\hline Missing & 596 & & & & & & \\
\hline \multicolumn{8}{|l|}{ Gestational weight gain } \\
\hline$<10 \mathrm{~kg}$ & 3,637 & 12.0 & 12.5 & 12.1 & 12.8 & 12.0 & 12.4 \\
\hline $10-15 \mathrm{~kg}$ & 13,837 & 45.6 & 44.4 & 42.3 & 41.6 & 45.6 & 8.2 \\
\hline $16-19 \mathrm{~kg}$ & 6,472 & 21.3 & 19.4 & 19.8 & 19.5 & 21.3 & 9.0 \\
\hline$\geq 20 \mathrm{~kg}$ & 6,422 & 21.2 & 26.7 & 25.8 & 26.0 & 21.2 & 12.1 \\
\hline Missing & 7,396 & & & & & & \\
\hline \multicolumn{8}{|l|}{ Smoking in pregnancy } \\
\hline None & 32,413 & 85.9 & 76.8 & 78.5 & 78.4 & 79.9 & 9.0 \\
\hline $0-10$ cigarettes/day & 4,208 & 11.1 & 17.4 & 16.5 & 16.5 & 15.4 & 14.6 \\
\hline$>10$ cigarettes/day & 1,103 & 3.0 & 5.8 & 5.0 & 5.1 & 4.7 & 19.3 \\
\hline Missing & 40 & & & & & & \\
\hline \multicolumn{8}{|l|}{ Socioeconomic status } \\
\hline Higher & 26,191 & 69.6 & 61.7 & 65.4 & 61.3 & 66.9 & 8.4 \\
\hline Middle & 10,302 & 27.4 & 32.5 & 30.2 & 32.9 & 28.7 & 12.9 \\
\hline Lower & 1,150 & 3.1 & 5.8 & 4.4 & 5.8 & 4.4 & 15.7 \\
\hline Missing & 121 & & & & & & \\
\hline \multicolumn{8}{|l|}{ Breastfeeding } \\
\hline None or $<14$ weeks & 8,629 & 28.2 & 37.4 & 32.8 & 33.4 & 30.1 & 12.4 \\
\hline $14-21$ weeks & 13,089 & 42.7 & 37.8 & 39.4 & 39.1 & 40.2 & 8.6 \\
\hline$\geq 22$ weeks & 8,908 & 29.1 & 27.8 & 27.8 & 27.5 & 29.7 & 8.7 \\
\hline Missing & 7,138 & & & & & & \\
\hline \multicolumn{8}{|l|}{ Sex-child } \\
\hline Boy & 19,343 & 51.2 & 51.3 & 50.2 & 51.3 & 51.3 & 8.7 \\
\hline Girl & 18,421 & 48.8 & 48.7 & 49.8 & 48.7 & 48.7 & 11.1 \\
\hline
\end{tabular}


Table 2: Continued.

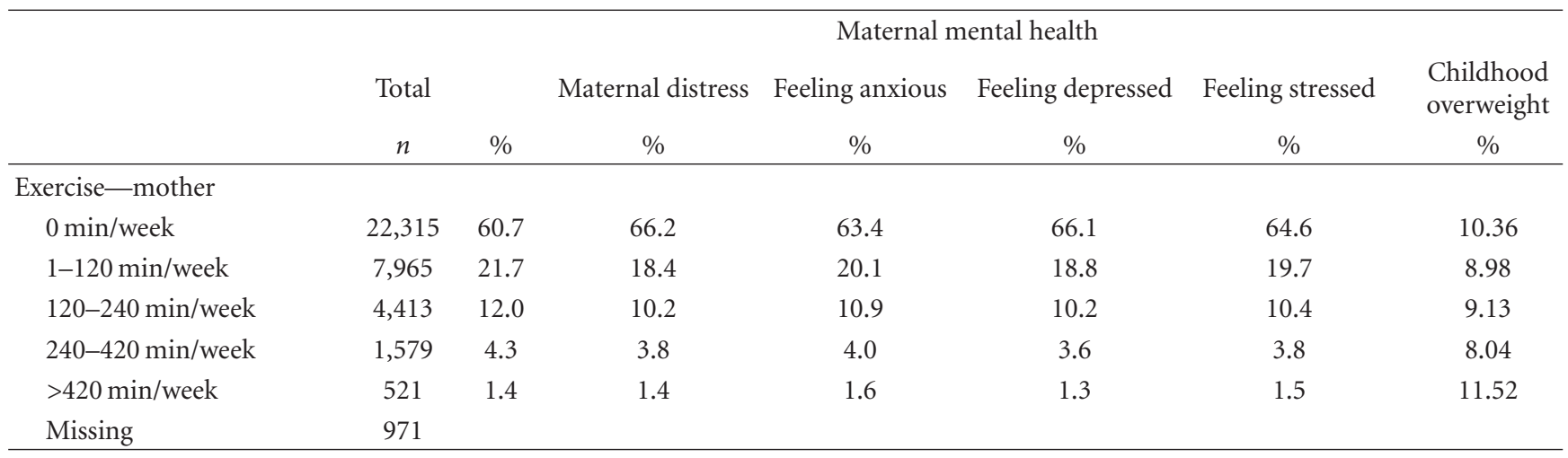

TABLE 3: Maternal distress during pregnancy and risk of childhood overweight at age 7.

\begin{tabular}{|c|c|c|c|c|c|c|c|c|c|}
\hline & \multicolumn{3}{|c|}{ All children } & \multicolumn{3}{|c|}{ Boys } & \multicolumn{3}{|c|}{ Girls } \\
\hline & & Crude OR & Adjusted $^{1}$ & & Crude OR & Adjusted $^{1}$ & Crude risk & Crude OR & Adjusted $^{1}$ \\
\hline & $\begin{array}{c}\text { Overweight } \\
\%\end{array}$ & $95 \%(\mathrm{CI})$ & $95 \%(\mathrm{CI})$ & $\begin{array}{c}\text { Overweight } \\
\%\end{array}$ & $95 \%(\mathrm{CI})$ & $95 \%(\mathrm{CI})$ & $\begin{array}{c}\text { Overweight } \\
\%\end{array}$ & $95 \%(\mathrm{CI})$ & $95 \%(\mathrm{CI})$ \\
\hline \multicolumn{10}{|c|}{ Maternal distress } \\
\hline A little & 9.63 & Ref. & Ref. & 8.4 & Ref. & Ref. & 11.1 & Ref. & Ref. \\
\hline A lot & 11.27 & 1.19 & $\begin{array}{c}1.06 \\
(0.96 ; 1.18)\end{array}$ & 10.7 & 1.30 & $\begin{array}{c}1.15 \\
(0.99 ; 1.33)\end{array}$ & 11.8 & 1.08 & $\begin{array}{c}0.98 \\
(0.85 ; 1.13)\end{array}$ \\
\hline \multicolumn{10}{|l|}{ Feeling anxious } \\
\hline A little & 9.73 & Ref. & Ref. & 8.5 & Ref. & Ref. & 11.1 & Ref. & Ref. \\
\hline A lot & 10.91 & 1.14 & $\begin{array}{c}1.06 \\
(0.95 ; 1.17)\end{array}$ & 10.7 & 1.29 & $\begin{array}{c}1.15 \\
(0.99 ; 1.34)\end{array}$ & 11.2 & 1.00 & $\begin{array}{c}0.97 \\
(0.84 ; 1.12)\end{array}$ \\
\hline \multicolumn{10}{|l|}{ Feeling depressed } \\
\hline A little & 9.61 & Ref. & Ref. & 8.4 & Ref. & Ref. & 10.9 & Ref. & Ref. \\
\hline A lot & 11.09 & 1.17 & $\begin{array}{c}1.02 \\
(0.94 ; 1.12)\end{array}$ & 10.2 & 1.24 & $\begin{array}{c}1.07 \\
(0.93 ; 1.21)\end{array}$ & 12.0 & 1.11 & $\begin{array}{c}0.99 \\
(0.87 ; 1.12)\end{array}$ \\
\hline \multicolumn{10}{|l|}{ Feeling stressed } \\
\hline A little & 9.65 & Ref. & Ref. & 8.4 & Ref. & Ref. & 11.0 & Ref. & Ref. \\
\hline A lot & 10.74 & 1.13 & $\begin{array}{c}1.05 \\
(0.97 ; 1.14)\end{array}$ & 9.9 & 1.20 & $\begin{array}{c}1.11 \\
(0.98 ; 1.25)\end{array}$ & 11.6 & 1.06 & $\begin{array}{c}1.00 \\
(0.89 ; 1.12)\end{array}$ \\
\hline \multicolumn{10}{|l|}{ Being worried } \\
\hline A little & 9.59 & Ref. & Ref. & 8.4 & Ref. & Ref. & 10.8 & Ref. & Ref. \\
\hline A lot & 11.55 & 1.23 & $\begin{array}{c}1.10 \\
(1.00 ; 1.22)\end{array}$ & 10.3 & 1.25 & $\begin{array}{c}1.10 \\
(0.96 ; 1.27)\end{array}$ & 12.8 & 1.21 & $\begin{array}{c}1.11 \\
(0.97 ; 1.26)\end{array}$ \\
\hline \multicolumn{10}{|l|}{$\begin{array}{l}\text { Lack of social } \\
\text { support }\end{array}$} \\
\hline No & 9.98 & Ref. & Ref. & 8.8 & Ref. & Ref. & 11.2 & Ref. & Ref. \\
\hline Yes & 8.60 & 0.85 & $\begin{array}{c}0.93 \\
(0.82 ; 1.04)\end{array}$ & 7.6 & 0.85 & $\begin{array}{c}0.92 \\
(0.78 ; 1.09)\end{array}$ & 9.6 & 0.85 & $\begin{array}{c}0.94 \\
(0.80 ; 1.10)\end{array}$ \\
\hline \multicolumn{10}{|l|}{$\begin{array}{l}\text { Socioeconomic } \\
\text { status }\end{array}$} \\
\hline High & 8.41 & Ref. & Ref. & 7.4 & Ref. & Ref. & 9.5 & Ref. & Ref. \\
\hline Intermediate & 12.92 & 1.61 & $\begin{array}{c}1.31 \\
(1.21 ; 1.42)\end{array}$ & 11.6 & 1.59 & $\begin{array}{c}1.28 \\
(1.14 ; 1.44)\end{array}$ & 14.3 & 1.59 & $\begin{array}{c}1.33 \\
(1.20 ; 1.48)\end{array}$ \\
\hline Low & 15.65 & 2.02 & $\begin{array}{c}1.49 \\
(1.25 ; 1.80)\end{array}$ & 12.8 & 1.84 & $\begin{array}{c}1.29 \\
(0.98 ; 1.69)\end{array}$ & 18.5 & 2.16 & $\begin{array}{c}1.66 \\
(1.32 ; 2.09)\end{array}$ \\
\hline
\end{tabular}

\footnotetext{
${ }^{1}$ Adjusted for age, parity, prepregnancy BMI, smoking during pregnancy and socioeconomic status.
} 
pregnancy and overweight of the children at 7 years of age is to some extent in agreement with this study.

Gender-specific effects may exist as hormonal changes during pregnancy due to maternal distress may lead to different expressions in boys and girls and result in different disease profiles [13]. Our study indicated a minor gender difference in the association between maternal distress and childhood overweight as boys had a modest increased risk and girls did not. However, this finding was not statistically significant and needs to be replicated in other data sources.

Children of mothers who tended to worry during pregnancy had a slightly increased risk of overweight. Possibly, the same mechanisms as with maternal distress are at play or it may be that these mothers are more protective of their children. However, a previous study in the same cohort of feelings of distress, covering anxiety, depression, and stress during the first 6-month postpartum did also not find an increased risk of childhood overweight [20]. Although parenting strategies may differ depending on levels of distress, it does not seem to be of clinical importance. Our results also indicate that children of women with lack of social support had a small decreased risk for overweight.

This study has several strengths and limitations that need to be addressed. The availability of the DNBC with its prospective design and detailed data collection presented a unique opportunity to study the associations between maternal distress during pregnancy and childhood overweight. Due to the large size of the cohort, the statistical precision was high so it should be possible to detect true relevant associations.

Our information on maternal distress relied on selfreported data. This may cause misclassification of the exposure, but since the mothers had no knowledge of the later weight status of their child at the time of the pregnancy interview this is not likely to cause serious bias and if so, would most likely underestimate the effect. The classification of distress is important as stressor-specific pathways may differ between the different types of stress [21]. Tegethoff et al. previously studied different types of stress in the same cohort and found emotional stress and life stress, associated with different types of diseases in the offspring [6]. We analyzed both combined and separate measures of maternal distress, which is measured like Tegethoff et al.'s measurement of emotional stress and found no association with overweight in the offspring. Neither did we find life stress to be associated with childhood overweight in separate analyses.

Childhood overweight of the child was based on selfreported information from the parents on weight and height of the child, who was measured either by the parents, the general practitioner or the school nurse. We expect some measurement error, but unrelated to the level of maternal distress during pregnancy and therefore it should not cause any serious misclassification bias, but only attenuate the associations under study. This was also supported by a validation study in 1200 children participating in the 7-year follow up, which found no systematic errors.

To reach the final study population, we excluded approximately $40 \%$ of the women who initially participated in the pregnancy interviews because they did not participate in the 7-year follow up. A recent study found that participants in the 7-year follow up were more often of high socioeconomic status and healthier [22]. We also confirmed this finding in sub analyses of our data and moreover found mothers who reported to be anxious, depressed, or stressed during pregnancy to be less likely to participate in the follow up. This selection may bias our results if it was also associated with childhood overweight. We have no data to investigate this, but believe this problem is minor as for similar exposureoutcome associations, based on participation in the 7-year follow up, such bias appeared to be small [22].

\section{Conclusion}

The etiology of childhood overweight is still poorly understood. In conclusion, this study in a generally healthy population of Scandinavian women did not provide evidence to the hypothesis that prenatal maternal distress causes childhood overweight. Further studies with more exact measures of psychosocial distress, continuous measures of distress, and in populations with higher stress levels are needed to perhaps find a threshold as to when more commonly experienced distress in the mother during pregnancy may cause childhood overweight and obesity.

\section{Abbreviations}

BMI: Body mass index

OR: Odds ratio

WHO: World Health Organization.

\section{Conflict of Interests}

The authors declare no conflict of interests.

\section{Acknowledgments}

The Danish National Research Foundation has established the Danish Epidemiology Science Centre that initiated and created the Danish National Birth Cohort. The cohort is furthermore a result of a major grant from this Foundation. Additional support for the Danish National Birth Cohort is obtained from the Pharmacy Foundation, the Egmont Foundation, the March of Dimes Birth Defects Foundation, the Augustinus Foundation, and the Health Foundation. The DNBC 7-year followup was supported by the Lundbeck Foundation (195/04) and the Danish Medical Research Council (SSVF 0646). The study is part of the activities in the Danish Obesity Research Centre (http://www.danorc.dk/).

\section{References}

[1] S. Pearson, L. W. Olsen, B. Hansen, and T. I. A. Sørensen, "Increase in overweight and obesity amongst Copenhagen schoolchildren, 1947-2003," Ugeskrift for Laeger, vol. 167, no. 2, pp. 158-162, 2005.

[2] P. D. Gluckman and M. A. Hanson, "Developmental and epigenetic pathways to obesity: an evolutionary-developmental 
perspective," International Journal of Obesity, vol. 32, no. 7, pp. S62-S71, 2008.

[3] J. P. Herman and W. E. Cullinan, "Neurocircuitry of stress: central control of the hypothalamo-pituitary-adrenocortical axis," Trends in Neurosciences, vol. 20, no. 2, pp. 78-84, 1997.

[4] R. Gitau, A. Cameron, N. M. Fisk, and V. Glover, "Fetal exposure to maternal cortisol," The Lancet, vol. 352, no. 9129, pp. 707-708, 1998.

[5] C. Obel, M. Hedegaard, T. B. Henriksen, N. J. Secher, J. Olsen, and S. Levine, "Stress and salivary cortisol during pregnancy," Psychoneuroendocrinology, vol. 30, no. 7, pp. 647-656, 2005.

[6] M. Tegethoff, N. Greene, J. Olsen, E. Schaffner, and G. Meinlschmidt, "Stress during pregnancy and offspring pediatric disease: a national cohort study," Environmental Health Perspectives, vol. 119, no. 11, pp. 1647-1652, 2011.

[7] J. Li, M. Vestergaard, C. Obel et al., "Prenatal stress and cerebral palsy: a nationwide cohort study in Denmark," Psychosomatic Medicine, vol. 71, no. 6, pp. 615-618, 2009.

[8] F. Fang, C. O. Hoglund, P. Arck et al., "Maternal bereavement and childhood asthma-analyses in two large samples of Swedish children," PLoS ONE, vol. 6, no. 11, Article ID e27202, 2011.

[9] J. Virk, J. Li, M. Vestergaard, C. Obel, M. Lu, and J. Olsen, "Early life disease programming during the preconception and prenatal period: making the link between stressful life events and type-1 diabetes," PLOS ONE, vol. 5, no. 7, Article ID e11523, 2010.

[10] J. Li, M. Vestergaard, C. Obel et al., "Prenatal stress and epilepsy in later life: a nationwide follow-up study in Denmark," Epilepsy Research, vol. 81, no. 1, pp. 52-57, 2008.

[11] J. Li, M. Vestergaard, C. Obel et al., "A nationwide study on the risk of autism after prenatal stress exposure to maternal bereavement," Pediatrics, vol. 123, no. 4, pp. 1102-1107, 2009.

[12] J. Li, J. Olsen, M. Vestergaard, C. Obel, J. L. Baker, and T. I. A. Sørensen, "Prenatal stress exposure related to maternal bereavement and risk of childhood overweight," PLoS ONE, vol. 5, no. 7, Article ID e11896, 2010.

[13] A. B. Wisniewski and S. D. Chernausek, "Gender in childhood obesity: family environment, hormones, and genes," Gender Medicine, vol. 6, no. 1, pp. 76-85, 2009.

[14] J. Olsen, M. Melbye, S. F. Olsen et al., "The Danish National Birth Cohort-its background, structure and aim," Scandinavian Journal of Public Health, vol. 29, no. 4, pp. 300-307, 2001.

[15] L. R. Olsen, E. L. Mortensen, and P. Bech, "The SCL-90 and SCL-90R versions validated by item response models in a Danish community sample," Acta Psychiatrica Scandinavica, vol. 110, no. 3, pp. 225-229, 2004.

[16] L. Derogatis, SCL-90-R Administration, Scoring and Procedures Manual-II, Clinical Psychometric Research, Towson, Md, USA, 2nd edition, 1992.

[17] G. Asessment, General Health Questionnaire, Frequently Asked Questions. 2007, http://www.gl-assessment.co.uk/ health_and_psychology/resources/general_health_questionnaire/faqs.asp?css $=0$-faq 1 .

[18] T. J. Cole, M. C. Bellizzi, K. M. Flegal, and W. H. Dietz, "Establishing a standard definition for child overweight and obesity worldwide: international survey," British Medical Journal, vol. 320, no. 7244, pp. 1240-1243, 2000.

[19] T. N. Jacobsen, E. A. Nohr, and M. Frydenberg, "Selection by socioeconomic factors into the Danish National Birth Cohort," European Journal of Epidemiology, vol. 25, no. 5, pp. 349-355, 2010.
[20] T. A. Ajslev, C. S. Andersen, K. G. Ingstrup, E. A. Nohr, and T. I. A. Sørensen, "Maternal postpartum distress and childhood overweight," PLoS ONE, vol. 5, no. 6, Article ID e11136, 2010.

[21] K. Pacák and M. Palkovits, "Stressor specificity of central neuroendocrine responses: implications for stress-related disorders," Endocrine Reviews, vol. 22, no. 4, pp. 502-548, 2001.

[22] N. Greene, S. Greenland, J. Olsen, and E. A. Nohr, "Estimating bias from loss to follow-up in the Danish National Birth Cohort," Epidemiology, vol. 22, no. 6, pp. 815-822, 2011. 


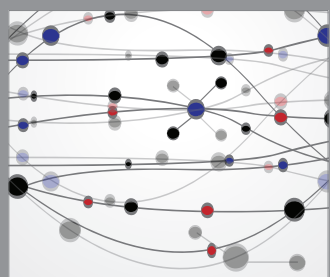

The Scientific World Journal
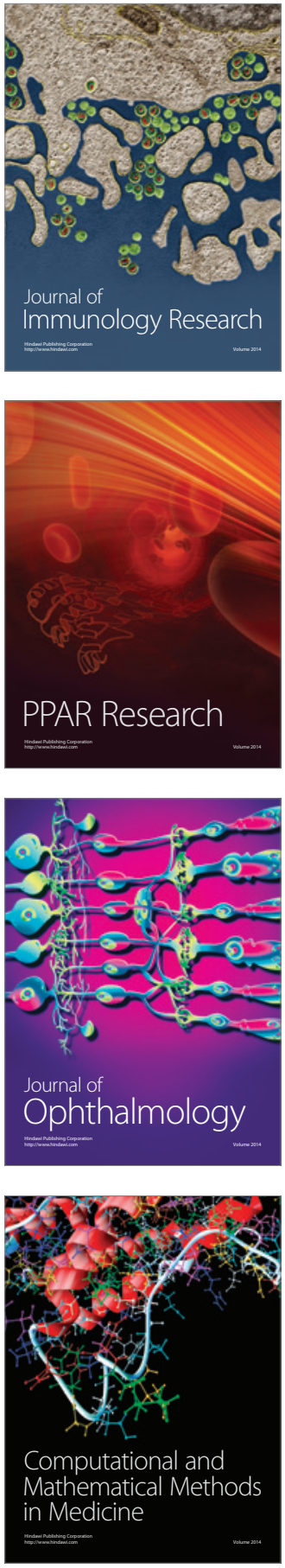

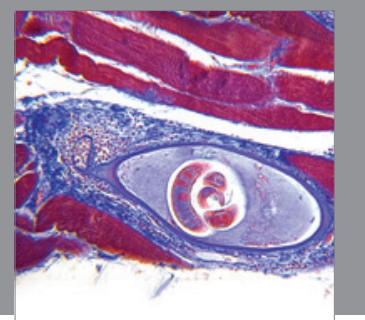

Gastroenterology

Research and Practice
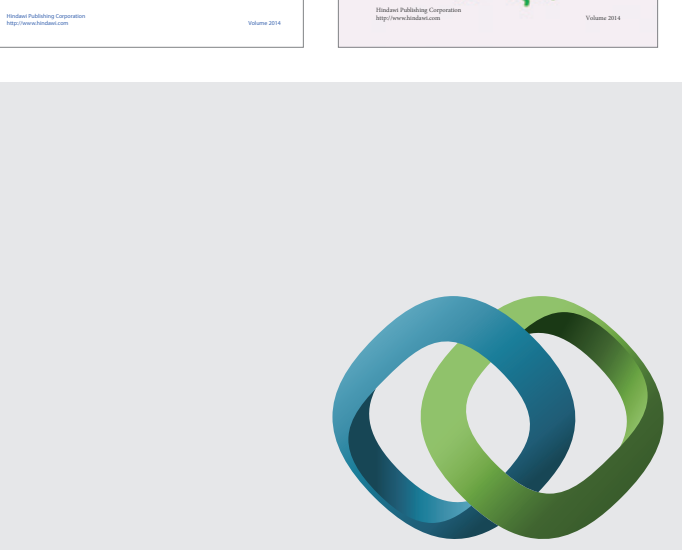

\section{Hindawi}

Submit your manuscripts at

http://www.hindawi.com
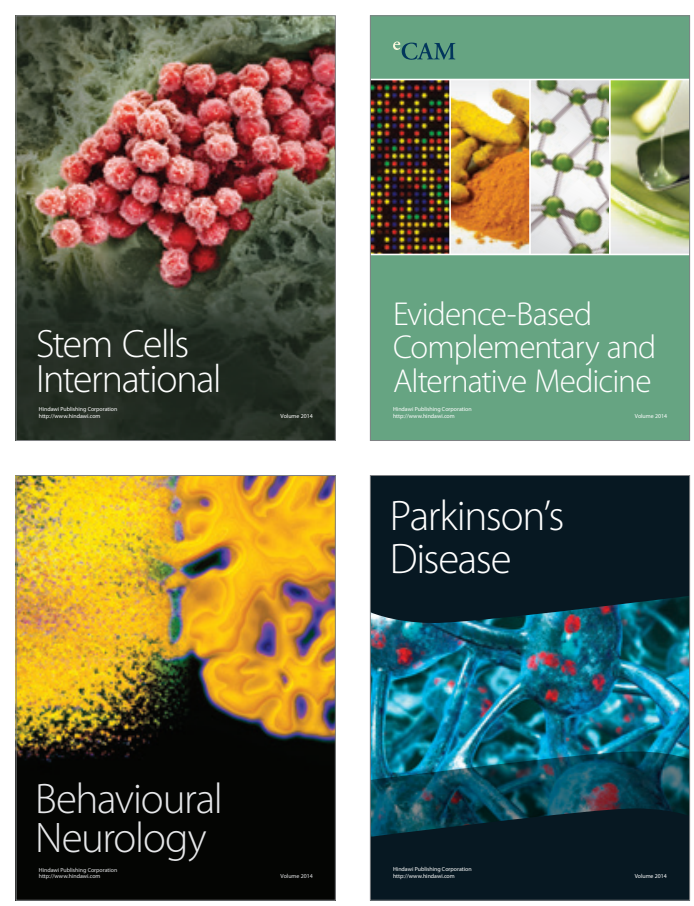

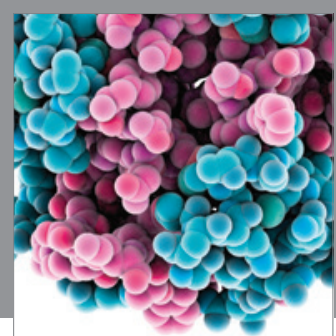

Journal of
Diabetes Research

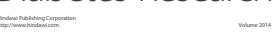

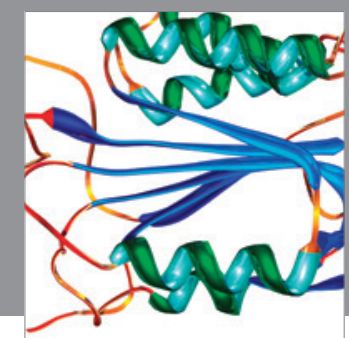

Disease Markers
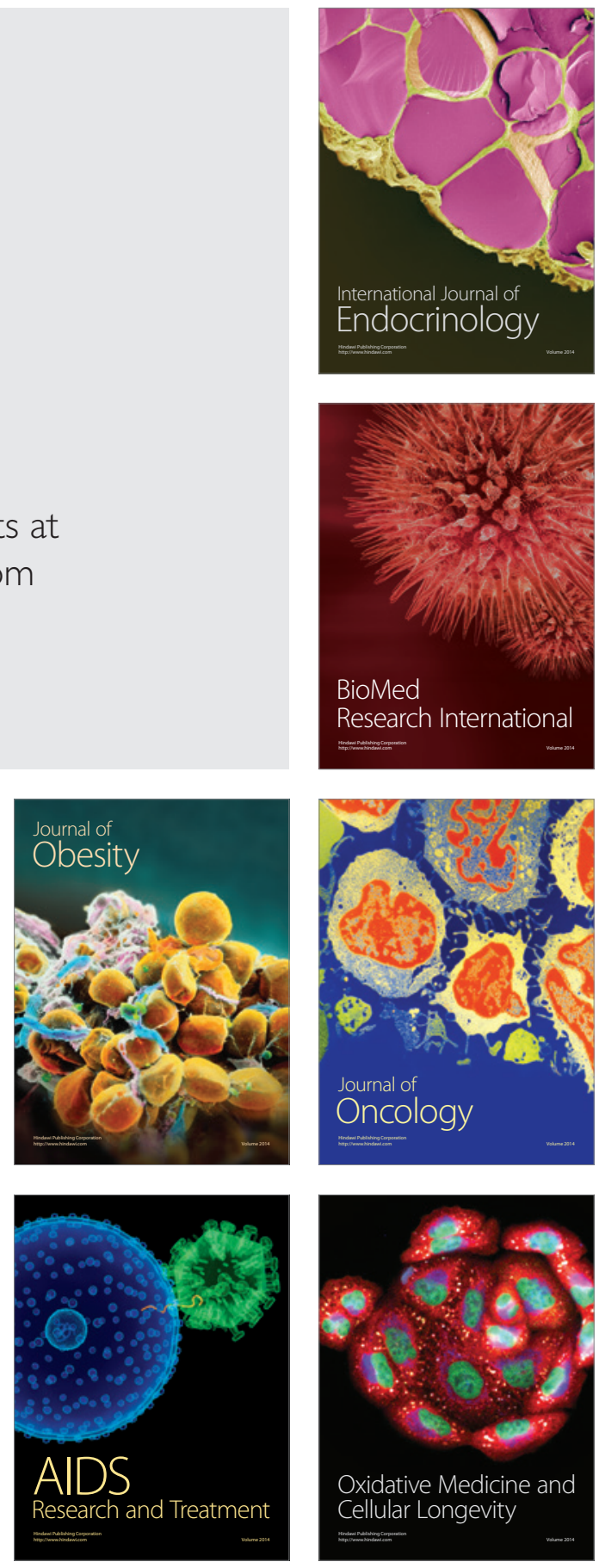